\title{
Design of digital filters for frequency weightings (A and C) required for risk assessments of workers exposed to noise
}

\author{
Andrew N. RIMELL ${ }^{1}$, Neil J. MANSFIELD ${ }^{2 *}$ and Gurmail S. PADDAN ${ }^{3}$ \\ ${ }^{1}$ Aero Engine Controls, Rolls-Royce, UK \\ ${ }^{2}$ Loughborough Design School, Loughborough University, UK \\ ${ }^{3}$ Institute of Naval Medicine, UK \\ Received January 4, 2013 and accepted August 18, 2014 \\ Published online in J-STAGE September 13, 2014
}

\begin{abstract}
Many workers are exposed to noise in their industrial environment. Excessive noise exposure can cause health problems and therefore it is important that the worker's noise exposure is assessed. This may require measurement by an equipment manufacturer or the employer. Human exposure to noise may be measured using microphones; however, weighting filters are required to correlate the physical noise sound pressure level measurements to the human's response to an auditory stimulus. IEC 61672-1 and ANSI S1.43 describe suitable weighting filters, but do not explain how to implement them for digitally recorded sound pressure level data. By using the bilinear transform, it is possible to transform the analogue equations given in the standards into digital filters. This paper describes the implementation of the weighting filters as digital IIR (Infinite Impulse Response) filters and provides all the necessary formulae to directly calculate the filter coefficients for any sampling frequency. Thus, the filters in the standards can be implemented in any numerical processing software (such as a spreadsheet or programming language running on a PC, mobile device or embedded system).
\end{abstract}

Key words: Hearing loss, IEC 61672-1, ANSI S1.43, Occupational health and safety, Noise measurement, Frequency weighting, Digital filter

\section{Introduction}

Humans are continuously exposed to noise as they go about their daily life, from household appliances through to transportation systems such as automobiles or trains. However, exposure to noise in the workplace can be a source of discomfort leading to health related problems such as temporary hearing loss, or even permanent hearing

*To whom correspondence should be addressed.

E-mail: n.j.mansfield@lboro.ac.uk

(C)2015 National Institute of Occupational Safety and Health loss if the correct protective measures are not in place. Limits on the amount of daily noise exposure to which workers may be exposed were tightened across Europe in $2003^{1)}$ and are being enforced by member states (for example, in the UK, the directive is being enforced through the Control of Noise at Work Regulations ${ }^{2)}$ ). Similar legislation has been introduced in the USA and Japan.

In order to assess the risk of noise to an operative, one course of action is to measure the noise level present in the operator's working environment. One problem for the equipment designer that exists in noise measurement is correlating the measured Sound Pressure Level (SPL) to the perceived loudness level of noise (ISO $226^{3}$ ). The A- 
and C-weightings (Fig. 1) are defined in IEC 61672-1 ${ }^{4)}$ and the equivalent ANSI S1.43-1997/2007 standard $^{5)}$. They are standardised for use in sound level meters and are required for the assessment of noise levels for legislative regulations such as the European Directive ${ }^{1)}$. The Aweighting is an approximation of the 40-Phon curve in ISO 226 and the $\mathrm{C}$-weighting is an approximation to the 100 -Phon curve. The A-weighting is a general purpose model of the human response to noise and used for environmental noise and hearing damage risk assessment. Both the $\mathrm{A}$-weighting and the $\mathrm{C}$-weighting are used to help in the specification of hearing protection through the use of the 'HML' method').

This paper takes the definitions of the A- and Cweightings and presents them in a format that can be used in a digital implementation without the need for specialist Digital Signal Processing knowledge. The paper takes the same form as one published previously by the authors where digital weighting filters were presented for assessment of workers exposed to whole-body and hand-arm vibration $^{7,8)}$.

\section{Standard Definition of the A- and C-Weightings}

There are three different frequency weighting filters described in IEC 61672-1: A, C and Z. As described above, the $\mathrm{A}$ - and $\mathrm{C}$-weightings are approximations to the equiloudness contours given in ISO 226. The Z-weighting is specified as having a flat frequency magnitude response of zero $\mathrm{dB}$, and is thus equivalent to not having a filter at all; therefore it will not be discussed any further in this paper. ANSI S1.43 describes three frequency weighting filters: A, $\mathrm{B}$ and $\mathrm{C}$; however only $\mathrm{A}$ and $\mathrm{C}$ are discussed in this paper because they are ones specified in the health legislation ${ }^{1)}$.

Both IEC 61672-1 and ANSI S1.43 present equations to calculate the frequency magnitude responses of the weighting filters. ANSI S1.42 ${ }^{9)}$ gives the equations required to calculate the analogue (s-domain) frequency response, impulse response and step response of both the A- and C-weightings. Equation 1 and Table 1 in ANSI $\mathrm{S} 1.42$ can be re-formatted to give the following s-domain equations:

$$
H_{A}(s)=G_{A} \frac{\omega_{4}^{2} \cdot s^{4}}{\left(s+\omega_{1}\right)^{2} \cdot\left(s+\omega_{2}\right) \cdot\left(s+\omega_{3}\right) \cdot\left(s+\omega_{4}\right)^{2}}
$$

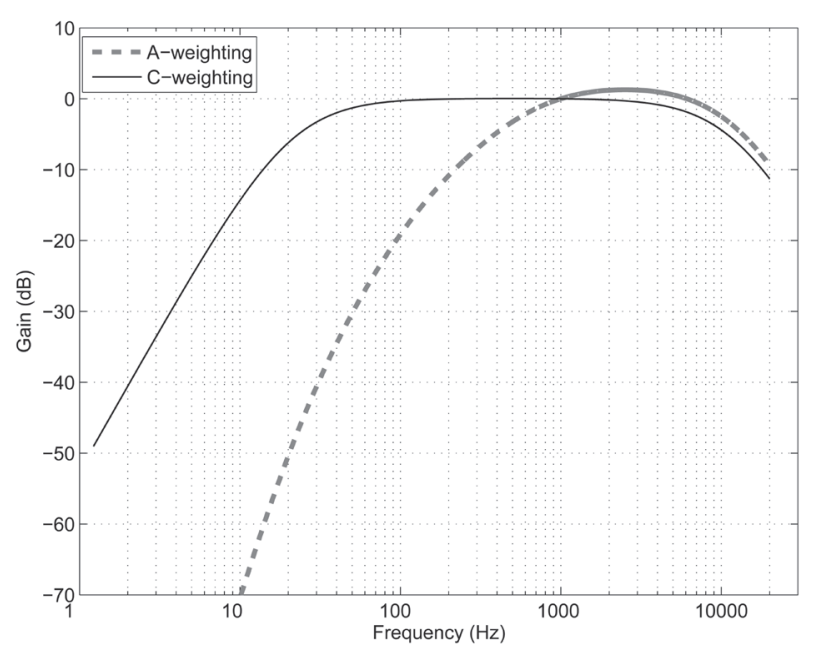

Fig. 1. Moduli of the $A$ and $C$ frequency weightings used in IEC 61672-1 and ANSI S1.43. Note that both weightings have $0 \mathrm{~dB}$ gain at $1 \mathrm{kHz}$.

$H_{C}(s)=G_{C} \frac{\omega_{4}{ }^{2} \cdot s^{4}}{\left(s+\omega_{1}\right)^{2} \cdot\left(s+\omega_{4}\right)^{2}}$

Where: $\omega=2 \cdot \pi \cdot f$ and $f_{1}=20.598997 \mathrm{~Hz}, f_{2}=107.65265 \mathrm{~Hz}$, $f_{3}=737.86223 \mathrm{~Hz}, f_{4}=12194.217 \mathrm{~Hz}$, and $G_{A}$ and $G_{C}$ are the gains (i.e. normalization constants) required to make the response equal to $0 \mathrm{~dB}$ at $1 \mathrm{kHz}$. The normalization constants are defined in IEC $61672-1$ as: $\mathrm{GA}=-2.0 \mathrm{~dB}$ and $\mathrm{GC}=0.062 \mathrm{~dB}$.

In IEC 61672-1 the frequencies are given as: $f_{1}=20.6 \mathrm{~Hz}, f_{2}=107.7 \mathrm{~Hz}, f_{3}=737.9 \mathrm{~Hz}$ and $f_{4}=12194.0 \mathrm{~Hz}$. Equations 1 and 2 are presented as analogue system equations and cannot directly be used in a digital (sampled) system. Firstly, the equations need to be converted from analogue (s-domain) to digital (z-domain).

\section{Design of Digital Weighting Filters Using the Bilinear Transform}

Modern data acquisition systems used for recording sound pressure level for analysis of human exposure are usually digital systems. Assuming a high quality digital recording has been obtained, the first stage of analysis using IEC $61672-1$ or ANSI S1.43 is to apply the weighting filter to the data. As the data are recorded in the timedomain, and subsequent processing is most straight forward if carried out in the time-domain, it is better to also perform filtering in the time-domain. Some metrics often quoted (e.g. peak pressure; r.m.s.) require a timedomain solution. An important parameter in the digital 
Table 1. Filter coefficients for the A-weighting filter

$\mathrm{a}[0] \quad 64+\left(16 \omega_{2}{ }^{\prime} \cdot \omega_{1}{ }^{\prime} \cdot \omega_{3}{ }^{\prime}\right)+\left(4 \omega_{2}{ }^{\prime} \cdot \omega_{1}{ }^{\prime 2} \cdot \omega_{3}{ }^{\prime}\right)+\left(32 \omega_{2}{ }^{\prime} \cdot \omega_{1}{ }^{\prime} \cdot \omega_{4}{ }^{\prime}\right)+\left(8 \omega_{2}{ }^{\prime} \cdot \omega_{1}{ }^{\prime 2} \cdot \omega_{4}{ }^{\prime}\right)+\left(32 \omega_{1}{ }^{\prime} \cdot \omega_{3}{ }^{\prime} \cdot \omega_{4}{ }^{\prime}\right)+\left(16 \omega_{2}{ }^{\prime} \cdot \omega_{3}{ }^{\prime} \cdot \omega_{4}{ }^{\prime}\right)+\left(64 \omega_{1}{ }^{\prime}\right)+\left(32 \omega_{2}{ }^{\prime}\right)+\left(32 \omega_{3}{ }^{\prime}\right)+(64$ $\left.\omega_{4}{ }^{\prime}\right)+\left(32 \omega_{2}{ }^{\prime} \cdot \omega_{1}{ }^{\prime}\right)+\left(8 \omega_{2}{ }^{\prime} \cdot \omega_{1}{ }^{2}\right)+\left(16 \omega_{1}{ }^{\prime 2}\right)+\left(16 \omega_{2}{ }^{\prime} \cdot \omega_{1}{ }^{\prime} \cdot \omega_{3}{ }^{\prime} \cdot \omega_{4}{ }^{\prime}\right)+\left(4 \omega_{2}{ }^{\prime} \cdot \omega_{1}{ }^{\prime 2} \cdot \omega_{3}{ }^{\prime} \cdot \omega_{4}{ }^{\prime}\right)+\left(32 \omega_{1}{ }^{\prime} \cdot \omega_{3}{ }^{\prime}\right)+\left(16 \omega_{2}{ }^{\prime} \cdot \omega_{3}{ }^{\prime}\right)+\left(8 \omega_{1}{ }^{\prime 2} \cdot \omega_{3}{ }^{\prime}\right)+\left(64 \omega_{1}{ }^{\prime} \cdot \omega_{4}{ }^{\prime}\right)+(32$ $\left.\omega_{2}{ }^{\prime} \cdot \omega_{4}{ }^{\prime}\right)+\left(32 \omega_{3}{ }^{\prime} \cdot \omega_{4}{ }^{\prime}\right)+\left(16 \omega_{1}{ }^{\prime 2} \cdot \omega_{4}{ }^{\prime}\right)+\left(8 \omega_{1}{ }^{\prime 2} \cdot \omega_{3}{ }^{\prime} \cdot \omega_{4}{ }^{\prime}\right)+\left(16 \omega_{4}{ }^{\prime 2}\right)+\left(16 \omega_{4}{ }^{\prime 2} \cdot \omega_{1}{ }^{\prime}\right)+\left(4 \omega_{4}{ }^{\prime 2} \cdot \omega_{1}{ }^{\prime 2}\right)+\left(4 \omega_{4}{ }^{\prime 2} \cdot \omega_{1}{ }^{\prime} \cdot \omega_{2}{ }^{\prime} \cdot \omega_{3}{ }^{\prime}\right)+\left(\omega_{4}{ }^{\prime 2} \cdot \omega_{1}{ }^{\prime 2} \cdot \omega_{2}{ }^{\prime} \cdot \omega_{3}{ }^{\prime}\right)+\left(8 \omega_{4}{ }^{\prime 2} \cdot\right.$ $\left.\omega_{1}{ }^{\prime} \cdot \omega_{2}{ }^{\prime}\right)+\left(2 \omega_{4}{ }^{\prime 2} \cdot \omega_{1}{ }^{\prime 2} \cdot \omega_{2}{ }^{\prime}\right)+\left(8 \omega_{4}{ }^{\prime 2} \cdot \omega_{2}{ }^{\prime}\right)+\left(8 \omega_{4}{ }^{\prime 2} \cdot \omega_{3}{ }^{\prime}\right)+\left(8 \omega_{4}{ }^{\prime 2} \cdot \omega_{1}{ }^{\prime} \cdot \omega_{3}{ }^{\prime}\right)+\left(2 \omega_{4}{ }^{\prime 2} \cdot \omega_{1}{ }^{\prime 2} \cdot \omega_{3}{ }^{\prime}\right)+\left(4 \omega_{4}{ }^{\prime 2} \cdot \omega_{2}{ }^{\prime} \cdot \omega_{3}{ }^{\prime}\right)$

$\mathrm{a}[1]-128+\left(64 \omega_{2}{ }^{\prime} \cdot \omega_{1}{ }^{\prime} \cdot \omega_{3}{ }^{\prime}\right)+\left(24 \omega_{2}{ }^{\prime} \cdot \omega_{1}{ }^{\prime 2} \cdot \omega_{3}{ }^{\prime}\right)+\left(128 \omega_{2}{ }^{\prime} \cdot \omega_{1}{ }^{\prime} \cdot \omega_{4}{ }^{\prime}\right)+\left(48 \omega_{2}{ }^{\prime} \cdot \omega_{1}{ }^{\prime 2} \cdot \omega_{4}{ }^{\prime}\right)+\left(128 \omega_{1}{ }^{\prime} \cdot \omega_{3}{ }^{\prime} \cdot \omega_{4}{ }^{\prime}\right)+\left(64 \omega_{2}{ }^{\prime} \cdot \omega_{3}{ }^{\prime} \cdot \omega_{4}{ }^{\prime}\right)+\left(64 \omega_{2}{ }^{\prime} \cdot \omega_{1}{ }^{\prime}\right)+\left(32 \omega_{2}{ }^{\prime} \cdot \omega_{1}{ }^{\prime 2}\right)$ $+\left(32 \omega_{1}{ }^{2}\right)+\left(96 \omega_{2}{ }^{\prime} \cdot \omega_{1}{ }^{\prime} \cdot \omega_{3}{ }^{\prime} \cdot \omega_{4}{ }^{\prime}\right)+\left(32 \omega_{2}{ }^{\prime} \cdot \omega_{1}{ }^{\prime 2} \cdot \omega_{3}{ }^{\prime} \cdot \omega_{4}{ }^{\prime}\right)+\left(64 \omega_{1}{ }^{\prime} \cdot \omega_{3}{ }^{\prime}\right)+\left(32 \omega_{2}{ }^{\prime} \cdot \omega_{3}{ }^{\prime}\right)+\left(32 \omega_{1}{ }^{\prime 2} \cdot \omega_{3}{ }^{\prime}\right)+\left(128 \omega_{1}{ }^{\prime} \cdot \omega_{4}{ }^{\prime}\right)+\left(64 \omega_{2}{ }^{\prime} \cdot \omega_{4}{ }^{\prime}\right)+\left(64 \omega_{3}{ }^{\prime} \cdot \omega_{4}{ }^{\prime}\right)+(64$ $\left.\omega_{1}{ }^{\prime 2} \cdot \omega_{4}{ }^{\prime}\right)+\left(48 \omega_{1}{ }^{\prime 2} \cdot \omega_{3}{ }^{\prime} \cdot \omega_{4}{ }^{\prime}\right)+\left(32 \omega_{4}{ }^{2}\right)+\left(64 \omega_{4}{ }^{\prime 2} \cdot \omega_{1}{ }^{\prime}\right)+\left(24 \omega_{4}{ }^{\prime 2} \cdot \omega_{1}{ }^{\prime 2}\right)+\left(32 \omega_{4}{ }^{\prime 2} \cdot \omega_{1}{ }^{\prime} \cdot \omega_{2}{ }^{\prime} \cdot \omega_{3}{ }^{\prime}\right)+\left(10 \omega_{4}{ }^{\prime 2} \cdot \omega_{1}{ }^{\prime 2} \cdot \omega_{2}{ }^{\prime} \cdot \omega_{3}{ }^{\prime}\right)+\left(48 \omega_{4}{ }^{\prime 2} \cdot \omega_{1}{ }^{\prime} \cdot \omega_{2}{ }^{\prime}\right)+\left(16 \omega_{4}{ }^{2} \cdot\right.$ $\left.\omega_{1}^{\prime 2} \cdot \omega_{2}{ }^{\prime}\right)+\left(32 \omega_{4}{ }^{\prime 2} \cdot \omega_{2}{ }^{\prime}\right)+\left(32 \omega_{4}{ }^{\prime 2} \cdot \omega_{3}{ }^{\prime}\right)+\left(48 \omega_{4}{ }^{\prime 2} \cdot \omega_{1}{ }^{\prime} \cdot \omega_{3}{ }^{\prime}\right)+\left(16 \omega_{4}{ }^{\prime 2} \cdot \omega_{1}{ }^{\prime 2} \cdot \omega_{3}{ }^{\prime}\right)+\left(24 \omega_{4}{ }^{\prime 2} \cdot \omega_{2}{ }^{\prime} \cdot \omega_{3}{ }^{\prime}\right)$

$\mathrm{a}[2]-192+\left(48 \omega_{2}{ }^{\prime} \cdot \omega_{1}{ }^{\prime} \cdot \omega_{3}{ }^{\prime}\right)+\left(52 \omega_{2}{ }^{\prime} \cdot \omega_{1}{ }^{2} \cdot \omega_{3}{ }^{\prime}\right)+\left(96 \omega_{2}{ }^{\prime} \cdot \omega_{1}{ }^{\prime} \cdot \omega_{4}{ }^{\prime}\right)+\left(104 \omega_{2}{ }^{\prime} \cdot \omega_{1}{ }^{\prime 2} \cdot \omega_{4}{ }^{\prime}\right)+\left(96 \omega_{1}{ }^{\prime} \cdot \omega_{3}{ }^{\prime} \cdot \omega_{4}{ }^{\prime}\right)+\left(48 \omega_{2}{ }^{\prime} \cdot \omega_{3}{ }^{\prime} \cdot \omega_{4}{ }^{\prime}\right)-\left(320 \omega_{1}{ }^{\prime}\right)-\left(160 \omega_{2}{ }^{\prime}\right)-(160$ $\left.\omega_{3}{ }^{\prime}\right)-\left(320 \omega_{4}{ }^{\prime}\right)-\left(96 \omega_{2}{ }^{\prime} \cdot \omega_{1}{ }^{\prime}\right)+\left(24 \omega_{2}{ }^{\prime} \cdot \omega_{1}{ }^{\prime 2}\right)-\left(48 \omega_{1}{ }^{\prime 2}\right)+\left(208 \omega_{2}{ }^{\prime} \cdot \omega_{1}{ }^{\prime} \cdot \omega_{3}{ }^{\prime} \cdot \omega_{4}{ }^{\prime}\right)+\left(108 \omega_{2}{ }^{\prime} \cdot \omega_{1}{ }^{\prime 2} \cdot \omega_{3}{ }^{\prime} \cdot \omega_{4}{ }^{\prime}\right)-\left(96 \omega_{1}{ }^{\prime} \cdot \omega_{3}{ }^{\prime}\right)-\left(48 \omega_{2}{ }^{\prime} \cdot \omega_{3}{ }^{\prime}\right)+\left(24 \omega_{1}{ }^{\prime 2} \cdot \omega_{3}{ }^{\prime}\right)-$ $\left(192 \omega_{1}{ }^{\prime} \cdot \omega_{4}{ }^{\prime}\right)-\left(96 \omega_{2}{ }^{\prime} \cdot \omega_{4}{ }^{\prime}\right)-\left(96 \omega_{3}{ }^{\prime} \cdot \omega_{4}{ }^{\prime}\right)+\left(48 \omega_{1}{ }^{\prime 2} \cdot \omega_{4}{ }^{\prime}\right)+\left(104 \omega_{1}{ }^{\prime 2} \cdot \omega_{3}{ }^{\prime} \cdot \omega_{4}{ }^{\prime}\right)-\left(48 \omega_{4}{ }^{2}\right)+\left(48 \omega_{4}{ }^{\prime 2} \cdot \omega_{1}{ }^{\prime}\right)+\left(52 \omega_{4}{ }^{\prime 2} \cdot \omega_{1}{ }^{\prime 2}\right)+\left(108 \omega_{4}{ }^{\prime 2} \cdot \omega_{1}{ }^{\prime} \cdot \omega_{2}{ }^{\prime} \cdot \omega_{3}{ }^{\prime}\right)+(45$ $\left.\omega_{4}{ }^{\prime 2} \cdot \omega_{1}^{\prime 2} \cdot \omega_{2}{ }^{\prime} \cdot \omega_{3}{ }^{\prime}\right)+\left(104 \omega_{4}{ }^{\prime 2} \cdot \omega_{1}{ }^{\prime} \cdot \omega_{2}{ }^{\prime}\right)+\left(54 \omega_{4}{ }^{\prime 2} \cdot \omega_{1}{ }^{\prime 2} \cdot \omega_{2}{ }^{\prime}\right)+\left(24 \omega_{4}{ }^{\prime 2} \cdot \omega_{2}{ }^{\prime}\right)+\left(24 \omega_{4}{ }^{\prime 2} \cdot \omega_{3}{ }^{\prime}\right)+\left(104 \omega_{4}{ }^{\prime 2} \cdot \omega_{1}{ }^{\prime} \cdot \omega_{3}{ }^{\prime}\right)+\left(54 \omega_{4}{ }^{\prime 2} \cdot \omega_{1}{ }^{\prime 2} \cdot \omega_{3}{ }^{\prime}\right)+\left(52 \omega_{4}{ }^{\prime 2} \cdot \omega_{2}{ }^{\prime} \cdot \omega_{3}{ }^{\prime}\right)$

a[3] $512-\left(128 \omega_{2}{ }^{\prime} \cdot \omega_{1}{ }^{\prime} \cdot \omega_{3}{ }^{\prime}\right)+\left(32 \omega_{2}{ }^{\prime} \cdot \omega_{1}{ }^{\prime 2} \cdot \omega_{3}{ }^{\prime}\right)-\left(256 \omega_{2}{ }^{\prime} \cdot \omega_{1}{ }^{\prime} \cdot \omega_{4}{ }^{\prime}\right)+\left(64 \omega_{2}{ }^{\prime} \cdot \omega_{1}{ }^{\prime 2} \cdot \omega_{4}{ }^{\prime}\right)-\left(256 \omega_{1}{ }^{\prime} \cdot \omega_{3}{ }^{\prime} \cdot \omega_{4}{ }^{\prime}\right)-\left(128 \omega_{2}{ }^{\prime} \cdot \omega_{3}{ }^{\prime} \cdot \omega_{4}{ }^{\prime}\right)-\left(256 \omega_{2}{ }^{\prime} \cdot \omega_{1}{ }^{\prime}\right)-\left(64 \omega_{2}{ }^{\prime} \cdot\right.$ $\left.\omega_{1}{ }^{\prime 2}\right)-\left(128 \omega_{1}{ }^{\prime 2}\right)+\left(128 \omega_{2}{ }^{\prime} \cdot \omega_{1}{ }^{\prime} \cdot \omega_{3}{ }^{\prime} \cdot \omega_{4}{ }^{\prime}\right)+\left(192 \omega_{2}{ }^{\prime} \cdot \omega_{1}{ }^{\prime 2} \cdot \omega_{3}{ }^{\prime} \cdot \omega_{4}{ }^{\prime}\right)-\left(256 \omega_{1}{ }^{\prime} \cdot \omega_{3}{ }^{\prime}\right)-\left(128 \omega_{2}{ }^{\prime} \cdot \omega_{3}{ }^{\prime}\right)-\left(64 \omega_{1}{ }^{\prime 2} \cdot \omega_{3}{ }^{\prime}\right)-\left(512 \omega_{1}{ }^{\prime} \cdot \omega_{4}{ }^{\prime}\right)-\left(256 \omega_{2}{ }^{\prime} \cdot \omega_{4}{ }^{\prime}\right)-\left(256 \omega_{3}{ }^{\prime}\right.$ $\left.\cdot \omega_{4}{ }^{\prime}\right)-\left(128 \omega_{1}{ }^{\prime 2} \cdot \omega_{4}{ }^{\prime}\right)+\left(64 \omega_{1}{ }^{\prime 2} \cdot \omega_{3}{ }^{\prime} \cdot \omega_{4}{ }^{\prime}\right)-\left(128 \omega_{4}{ }^{\prime 2}\right)-\left(128 \omega_{4}{ }^{\prime 2} \cdot \omega_{1}{ }^{\prime}\right)+\left(32 \omega_{4}{ }^{\prime 2} \cdot \omega_{1}{ }^{\prime 2}\right)+\left(192 \omega_{4}{ }^{\prime 2} \cdot \omega_{1}{ }^{\prime} \cdot \omega_{2}{ }^{\prime} \cdot \omega_{3}{ }^{\prime}\right)+\left(120 \omega_{4}{ }^{\prime 2} \cdot \omega_{1}{ }^{\prime 2} \cdot \omega_{2}{ }^{\prime} \cdot \omega_{3}{ }^{\prime}\right)+\left(64 \omega_{4}{ }^{\prime 2} \cdot \omega_{1}{ }^{\prime} \cdot\right.$ $\left.\omega_{2}{ }^{\prime}\right)+\left(96 \omega_{4}{ }^{\prime 2} \cdot \omega_{1}{ }^{\prime 2} \cdot \omega_{2}{ }^{\prime}\right)-\left(64 \omega_{4}{ }^{\prime 2} \cdot \omega_{2}{ }^{\prime}\right)-\left(64 \omega_{4}{ }^{\prime 2} \cdot \omega_{3}{ }^{\prime}\right)+\left(64 \omega_{4}{ }^{\prime 2} \cdot \omega_{1}{ }^{\prime} \cdot \omega_{3}{ }^{\prime}\right)+\left(96 \omega_{4}{ }^{\prime 2} \cdot \omega_{1}{ }^{\prime 2} \cdot \omega_{3}{ }^{\prime}\right)+\left(32 \omega_{4}{ }^{\prime 2} \cdot \omega_{2}{ }^{\prime} \cdot \omega_{3}{ }^{\prime}\right)$

a[4] $128-\left(224 \omega_{2}{ }^{\prime} \cdot \omega_{1}{ }^{\prime} \cdot \omega_{3}{ }^{\prime}\right)-\left(56 \omega_{2}{ }^{\prime} \cdot \omega_{1}{ }^{\prime 2} \cdot \omega_{3}{ }^{\prime}\right)-\left(448 \omega_{2}{ }^{\prime} \cdot \omega_{1}{ }^{\prime} \cdot \omega_{4}{ }^{\prime}\right)-\left(112 \omega_{2}{ }^{\prime} \cdot \omega_{1}{ }^{\prime 2} \cdot \omega_{4}{ }^{\prime}\right)-\left(448 \omega_{1}{ }^{\prime} \cdot \omega_{3}{ }^{\prime} \cdot \omega_{4}{ }^{\prime}\right)-\left(224 \omega_{2}{ }^{\prime} \cdot \omega_{3}{ }^{\prime} \cdot \omega_{4}{ }^{\prime}\right)+\left(640 \omega_{1}{ }^{\prime}\right)+\left(320 \omega_{2}{ }^{\prime}\right)+(320$ $\left.\omega_{3}{ }^{\prime}\right)+\left(640 \omega_{4}{ }^{\prime}\right)+\left(64 \omega_{2}{ }^{\prime} \cdot \omega_{1}{ }^{\prime}\right)-\left(112 \omega_{2}{ }^{\prime} \cdot \omega_{1}{ }^{\prime 2}\right)+\left(32 \omega_{1}{ }^{\prime 2}\right)-\left(224 \omega_{2}{ }^{\prime} \cdot \omega_{1}{ }^{\prime} \cdot \omega_{3}{ }^{\prime} \cdot \omega_{4}{ }^{\prime}\right)+\left(168 \omega_{2}{ }^{\prime} \cdot \omega_{1}{ }^{\prime 2} \cdot \omega_{3}{ }^{\prime} \cdot \omega_{4}{ }^{\prime}\right)+\left(64 \omega_{1}{ }^{\prime} \cdot \omega_{3}{ }^{\prime}\right)+\left(32 \omega_{2}{ }^{\prime} \cdot \omega_{3}{ }^{\prime}\right)-\left(112 \omega_{1}{ }^{\prime 2} \cdot \omega_{3}{ }^{\prime}\right)+$ $\left(128 \omega_{1}{ }^{\prime} \cdot \omega_{4}{ }^{\prime}\right)+\left(64 \omega_{2}{ }^{\prime} \cdot \omega_{4}{ }^{\prime}\right)+\left(64 \omega_{3}{ }^{\prime} \cdot \omega_{4}{ }^{\prime}\right)-\left(224 \omega_{1}{ }^{\prime 2} \cdot \omega_{4}{ }^{\prime}\right)-\left(112 \omega_{1}{ }^{\prime 2} \cdot \omega_{3}{ }^{\prime} \cdot \omega_{4}{ }^{\prime}\right)+\left(32 \omega_{4}{ }^{2}\right)-\left(224 \omega_{4}{ }^{\prime 2} \cdot \omega_{1}{ }^{\prime}\right)-\left(56 \omega_{4}{ }^{\prime 2} \cdot \omega_{1}{ }^{\prime 2}\right)+\left(168 \omega_{4}{ }^{\prime 2} \cdot \omega_{1}{ }^{\prime} \cdot \omega_{2}{ }^{\prime} \cdot \omega_{3}{ }^{\prime}\right)+(210$ $\left.\omega_{4}{ }^{\prime 2} \cdot \omega_{1}{ }^{\prime 2} \cdot \omega_{2}{ }^{\prime} \cdot \omega_{3}{ }^{\prime}\right)-\left(112 \omega_{4}{ }^{\prime 2} \cdot \omega_{1}{ }^{\prime} \cdot \omega_{2}{ }^{\prime}\right)+\left(84 \omega_{4}{ }^{\prime 2} \cdot \omega_{1}{ }^{\prime 2} \cdot \omega_{2}{ }^{\prime}\right)-\left(112 \omega_{4}{ }^{\prime 2} \cdot \omega_{2}{ }^{\prime}\right)-\left(112 \omega_{4}{ }^{\prime 2} \cdot \omega_{3}{ }^{\prime}\right)-\left(112 \omega_{4}{ }^{\prime 2} \cdot \omega_{1}{ }^{\prime} \cdot \omega_{3}{ }^{\prime}\right)+\left(84 \omega_{4}{ }^{\prime 2} \cdot \omega_{1}{ }^{\prime 2} \cdot \omega_{3}{ }^{\prime}\right)-\left(56 \omega_{4}{ }^{\prime 2} \cdot \omega_{2}{ }^{\prime} \cdot \omega_{3}{ }^{\prime}\right)$

$\mathrm{a}[5]-\left(448 \omega_{2}{ }^{\prime} \cdot \omega_{1}{ }^{\prime} \cdot \omega_{3}{ }^{\prime} \cdot \omega_{4}{ }^{\prime}\right)-\left(224 \omega_{1}{ }^{\prime 2} \cdot \omega_{3}{ }^{\prime} \cdot \omega_{4}{ }^{\prime}\right)+\left(384 \omega_{3}{ }^{\prime} \cdot \omega_{4}{ }^{\prime}\right)-\left(112 \omega_{2}{ }^{\prime} \cdot \omega_{1}{ }^{\prime 2} \cdot \omega_{3}{ }^{\prime}\right)-\left(112 \omega_{4}{ }^{\prime 2} \cdot \omega_{1}{ }^{\prime 2}\right)+\left(384 \omega_{1}{ }^{\prime} \cdot \omega_{3}{ }^{\prime}\right)-\left(224 \omega_{4}{ }^{\prime 2} \cdot \omega_{1}{ }^{\prime} \cdot \omega_{3}{ }^{\prime}\right)+\left(192 \omega_{2}{ }^{\prime} \cdot \omega_{3}{ }^{\prime}\right)$ $-\left(224 \omega_{2}{ }^{\prime} \cdot \omega_{1}{ }^{\prime 2} \cdot \omega_{4}{ }^{\prime}\right)+\left(192 \omega_{1}{ }^{\prime 2}\right)+\left(252 \omega_{4}{ }^{\prime 2} \cdot \omega_{1}{ }^{\prime 2} \cdot \omega_{2}{ }^{\prime} \cdot \omega_{3}{ }^{\prime}\right)+\left(384 \omega_{2}{ }^{\prime} \cdot \omega_{1}{ }^{\prime}\right)-(768)-\left(224 \omega_{4}{ }^{\prime 2} \cdot \omega_{1}{ }^{\prime} \cdot \omega_{2}{ }^{\prime}\right)-\left(112 \omega_{4}{ }^{\prime 2} \cdot \omega_{2}{ }^{\prime} \cdot \omega_{3}{ }^{\prime}\right)+\left(384 \omega_{2}{ }^{\prime} \cdot \omega_{4}{ }^{\prime}\right)+\left(192 \omega_{4}{ }^{\prime 2}\right)+$ $\left(768 \omega_{1}{ }^{\prime} \cdot \omega_{4}{ }^{\prime}\right)$

a[6] $128+\left(224 \omega_{2}{ }^{\prime} \cdot \omega_{1}{ }^{\prime} \cdot \omega_{3}{ }^{\prime}\right)-\left(56 \omega_{2}{ }^{\prime} \cdot \omega_{1}{ }^{\prime 2} \cdot \omega_{3}{ }^{\prime}\right)+\left(448 \omega_{2}{ }^{\prime} \cdot \omega_{1}{ }^{\prime} \cdot \omega_{4}{ }^{\prime}\right)-\left(112 \omega_{2}{ }^{\prime} \cdot \omega_{1}{ }^{\prime 2} \cdot \omega_{4}{ }^{\prime}\right)+\left(448 \omega_{1}{ }^{\prime} \cdot \omega_{3}{ }^{\prime} \cdot \omega_{4}{ }^{\prime}\right)+\left(224 \omega_{2}{ }^{\prime} \cdot \omega_{3}{ }^{\prime} \cdot \omega_{4}{ }^{\prime}\right)-\left(640 \omega_{1}{ }^{\prime}\right)-\left(320 \omega_{2}{ }^{\prime}\right)-(320$ $\left.\omega_{3}{ }^{\prime}\right)-\left(640 \omega_{4}{ }^{\prime}\right)+\left(64 \omega_{2}{ }^{\prime} \cdot \omega_{1}{ }^{\prime}\right)+\left(112 \omega_{2}{ }^{\prime} \cdot \omega_{1}{ }^{\prime 2}\right)+\left(32 \omega_{1}{ }^{\prime 2}\right)-\left(224 \omega_{2}{ }^{\prime} \cdot \omega_{1}{ }^{\prime} \cdot \omega_{3}{ }^{\prime} \cdot \omega_{4}{ }^{\prime}\right)-\left(168 \omega_{2}{ }^{\prime} \cdot \omega_{1}{ }^{\prime 2} \cdot \omega_{3}{ }^{\prime} \cdot \omega_{4}{ }^{\prime}\right)+\left(64 \omega_{1}{ }^{\prime} \cdot \omega_{3}{ }^{\prime}\right)+\left(32 \omega_{2}{ }^{\prime} \cdot \omega_{3}{ }^{\prime}\right)+\left(112 \omega_{1}{ }^{\prime 2} \cdot \omega_{3}{ }^{\prime}\right)+$ $\left(128 \omega_{1}{ }^{\prime} \cdot \omega_{4}{ }^{\prime}\right)+\left(64 \omega_{2}{ }^{\prime} \cdot \omega_{4}{ }^{\prime}\right)+\left(64 \omega_{3}{ }^{\prime} \cdot \omega_{4}{ }^{\prime}\right)+\left(224 \omega_{1}{ }^{\prime 2} \cdot \omega_{4}{ }^{\prime}\right)-\left(112 \omega_{1}{ }^{\prime 2} \cdot \omega_{3}{ }^{\prime} \cdot \omega_{4}{ }^{\prime}\right)+\left(32 \omega_{4}{ }^{\prime 2}\right)+\left(224 \omega_{4}{ }^{\prime 2} \cdot \omega_{1}{ }^{\prime}\right)-\left(56 \omega_{4}{ }^{\prime 2} \cdot \omega_{1}{ }^{\prime 2}\right)-\left(168 \omega_{4}{ }^{\prime 2} \cdot \omega_{1}{ }^{\prime} \cdot \omega_{2}{ }^{\prime} \cdot \omega_{3}{ }^{\prime}\right)+(210$ $\left.\omega_{4}{ }^{\prime 2} \cdot \omega_{1}{ }^{\prime 2} \cdot \omega_{2}{ }^{\prime} \cdot \omega_{3}{ }^{\prime}\right)-\left(112 \omega_{4}{ }^{\prime 2} \cdot \omega_{1}{ }^{\prime} \cdot \omega_{2}{ }^{\prime}\right)-\left(84 \omega_{4}{ }^{\prime 2} \cdot \omega_{1}{ }^{\prime 2} \cdot \omega_{2}{ }^{\prime}\right)+\left(112 \omega_{4}{ }^{\prime 2} \cdot \omega_{2}{ }^{\prime}\right)+\left(112 \omega_{4}{ }^{\prime 2} \cdot \omega_{3}{ }^{\prime}\right)-\left(112 \omega_{4}{ }^{\prime 2} \cdot \omega_{1}{ }^{\prime} \cdot \omega_{3}{ }^{\prime}\right)-\left(84 \omega_{4}{ }^{\prime 2} \cdot \omega_{1}{ }^{\prime 2} \cdot \omega_{3}{ }^{\prime}\right)-\left(56 \omega_{4}{ }^{\prime 2} \cdot \omega_{2}{ }^{\prime} \cdot \omega_{3}{ }^{\prime}\right)$

a[7] $512+\left(128 \omega_{2}{ }^{\prime} \cdot \omega_{1}{ }^{\prime} \cdot \omega 3^{\prime}\right)+\left(32 \omega_{2}{ }^{\prime} \cdot \omega_{1}{ }^{\prime 2} \cdot \omega_{3}{ }^{\prime}\right)+\left(256 \omega_{2}{ }^{\prime} \cdot \omega_{1}{ }^{\prime} \cdot \omega_{4}{ }^{\prime}\right)+\left(64 \omega_{2}{ }^{\prime} \cdot \omega_{1}{ }^{\prime 2} \cdot \omega_{4}{ }^{\prime}\right)+\left(256 \omega_{1}{ }^{\prime} \cdot \omega_{3}{ }^{\prime} \cdot \omega_{4}{ }^{\prime}\right)+\left(128 \omega_{2}{ }^{\prime} \cdot \omega_{3}{ }^{\prime} \cdot \omega_{4}{ }^{\prime}\right)-\left(256 \omega_{2}{ }^{\prime} \cdot \omega_{1}{ }^{\prime}\right)+\left(64 \omega_{2}{ }^{\prime}\right.$. $\left.\omega_{1}{ }^{\prime 2}\right)-\left(128 \omega_{1}{ }^{\prime 2}\right)+\left(128 \omega_{2}{ }^{\prime} \cdot \omega_{1}{ }^{\prime} \cdot \omega_{3}{ }^{\prime} \cdot \omega_{4}{ }^{\prime}\right)-\left(192 \omega_{2}{ }^{\prime} \cdot \omega_{1}{ }^{\prime 2} \cdot \omega_{3}{ }^{\prime} \cdot \omega_{4}{ }^{\prime}\right)-\left(256 \omega_{1}{ }^{\prime} \cdot \omega_{3}{ }^{\prime}\right)-\left(128 \omega_{2}{ }^{\prime} \cdot \omega_{3}{ }^{\prime}\right)+\left(64 \omega_{1}{ }^{\prime 2} \cdot \omega_{3}{ }^{\prime}\right)-\left(512 \omega_{1}{ }^{\prime} \cdot \omega_{4}{ }^{\prime}\right)-\left(256 \omega_{2}{ }^{\prime} \cdot \omega_{4}{ }^{\prime}\right)-\left(256 \omega_{3}{ }^{\prime}\right.$ $\left.\cdot \omega_{4}{ }^{\prime}\right)+\left(128 \omega_{1}{ }^{\prime 2} \cdot \omega_{4}{ }^{\prime}\right)+\left(64 \omega_{1}{ }^{\prime 2} \cdot \omega_{3}{ }^{\prime} \cdot \omega_{4}{ }^{\prime}\right)-\left(128 \omega_{4}{ }^{\prime 2}\right)+\left(128 \omega_{4}{ }^{\prime 2} \cdot \omega_{1}{ }^{\prime}\right)+\left(32 \omega_{4}{ }^{\prime 2} \cdot \omega_{1}{ }^{\prime 2}\right)-\left(192 \omega_{4}{ }^{\prime 2} \cdot \omega_{1}{ }^{\prime} \cdot \omega_{2}{ }^{\prime} \cdot \omega_{3}{ }^{\prime}\right)+\left(120 \omega_{4}{ }^{\prime 2} \cdot \omega_{1}{ }^{\prime 2} \cdot \omega_{2}{ }^{\prime} \cdot \omega_{3}{ }^{\prime}\right)+\left(64 \omega_{4}{ }^{\prime 2} \cdot \omega_{1}{ }^{\prime} \cdot\right.$ $\left.\omega_{2}{ }^{\prime}\right)-\left(96 \omega_{4}{ }^{\prime 2} \cdot \omega_{1}{ }^{\prime 2} \cdot \omega_{2}{ }^{\prime}\right)+\left(64 \omega_{4}{ }^{\prime 2} \cdot \omega_{2}{ }^{\prime}\right)+\left(64 \omega_{4}{ }^{\prime 2} \cdot \omega_{3}{ }^{\prime}\right)+\left(64 \omega_{4}{ }^{\prime 2} \cdot \omega_{1}{ }^{\prime} \cdot \omega_{3}{ }^{\prime}\right)-\left(96 \omega_{4}{ }^{\prime 2} \cdot \omega_{1}{ }^{\prime 2} \cdot \omega_{3}{ }^{\prime}\right)+\left(32 \omega_{4}{ }^{\prime 2} \cdot \omega_{2}{ }^{\prime} \cdot \omega_{3}{ }^{\prime}\right)$

$\mathrm{a}[8]-192-\left(48 \omega_{2}{ }^{\prime} \cdot \omega_{1}{ }^{\prime} \cdot \omega_{3}{ }^{\prime}\right)+\left(52 \omega_{2}{ }^{\prime} \cdot \omega_{1}{ }^{\prime 2} \cdot \omega_{3}{ }^{\prime}\right)-\left(96 \omega_{2}{ }^{\prime} \cdot \omega_{1}{ }^{\prime} \cdot \omega_{4}{ }^{\prime}\right)+\left(104 \omega_{2}{ }^{\prime} \cdot \omega_{1}{ }^{\prime 2} \cdot \omega_{4}{ }^{\prime}\right)-\left(96 \omega_{1}{ }^{\prime} \cdot \omega_{3}{ }^{\prime} \cdot \omega_{4}{ }^{\prime}\right)-\left(48 \omega_{2}{ }^{\prime} \cdot \omega_{3}{ }^{\prime} \cdot \omega_{4}{ }^{\prime}\right)+\left(320 \omega_{1}{ }^{\prime}\right)+\left(160 \omega_{2}{ }^{\prime}\right)+(160$ $\left.\omega_{3}{ }^{\prime}\right)+\left(320 \omega_{4}{ }^{\prime}\right)-\left(96 \omega_{2}{ }^{\prime} \cdot \omega_{1}{ }^{\prime}\right)-\left(24 \omega_{2}{ }^{\prime} \cdot \omega_{1}{ }^{\prime 2}\right)-\left(48 \omega_{1}{ }^{\prime 2}\right)+\left(208 \omega_{2}{ }^{\prime} \cdot \omega_{1}{ }^{\prime} \cdot \omega_{3}{ }^{\prime} \cdot \omega_{4}{ }^{\prime}\right)-\left(108 \omega_{2}{ }^{\prime} \cdot \omega_{1}{ }^{\prime 2} \cdot \omega_{3}{ }^{\prime} \cdot \omega_{4}{ }^{\prime}\right)-\left(96 \omega_{1}{ }^{\prime} \cdot \omega_{3}{ }^{\prime}\right)-\left(48 \omega_{2}{ }^{\prime} \cdot \omega_{3}{ }^{\prime}\right)-\left(24 \omega_{1}{ }^{\prime 2} \cdot \omega_{3}{ }^{\prime}\right)-$ $\left(192 \omega_{1}{ }^{\prime} \cdot \omega_{4}{ }^{\prime}\right)-\left(96 \omega_{2}{ }^{\prime} \cdot \omega_{4}{ }^{\prime}\right)-\left(96 \omega_{3}{ }^{\prime} \cdot \omega_{4}{ }^{\prime}\right)-\left(48 \omega_{1}{ }^{\prime 2} \cdot \omega_{4}{ }^{\prime}\right)+\left(104 \omega_{1}{ }^{\prime 2} \cdot \omega_{3}{ }^{\prime} \cdot \omega_{4}{ }^{\prime}\right)-\left(48 \omega_{4}{ }^{\prime 2}\right)-\left(48 \omega_{4}{ }^{\prime 2} \cdot \omega_{1}{ }^{\prime}\right)+\left(52 \omega_{4}{ }^{\prime 2} \cdot \omega_{1}{ }^{\prime 2}\right)-\left(108 \omega_{4}{ }^{\prime 2} \cdot \omega_{1}{ }^{\prime} \cdot \omega_{2}{ }^{\prime} \cdot \omega_{3}{ }^{\prime}\right)+(45$ $\left.\omega_{4}{ }^{\prime 2} \cdot \omega_{1}{ }^{\prime 2} \cdot \omega_{2}{ }^{\prime} \cdot \omega_{3}{ }^{\prime}\right)+\left(104 \omega_{4}{ }^{\prime 2} \cdot \omega_{1}{ }^{\prime} \cdot \omega_{2}{ }^{\prime}\right)-\left(54 \omega_{4}{ }^{\prime 2} \cdot \omega_{1}{ }^{\prime 2} \cdot \omega_{2}{ }^{\prime}\right)-\left(24 \omega_{4}{ }^{\prime 2} \cdot \omega_{2}{ }^{\prime}\right)-\left(24 \omega_{4}{ }^{\prime 2} \cdot \omega_{3}{ }^{\prime}\right)+\left(104 \omega_{4}{ }^{\prime 2} \cdot \omega_{1}{ }^{\prime} \cdot \omega_{3}{ }^{\prime}\right)-\left(54 \omega_{4}{ }^{\prime 2} \cdot \omega_{1}{ }^{\prime 2} \cdot \omega_{3}{ }^{\prime}\right)+\left(52 \omega_{4}{ }^{\prime 2} \cdot \omega_{2}{ }^{\prime} \cdot \omega_{3}{ }^{\prime}\right)$

$\mathrm{a}[9]-128-\left(64 \omega_{2}{ }^{\prime} \cdot \omega_{1}{ }^{\prime} \cdot \omega_{3}{ }^{\prime}\right)+\left(24 \omega_{2}{ }^{\prime} \cdot \omega_{1}{ }^{\prime 2} \cdot \omega_{3}{ }^{\prime}\right)-\left(128 \omega_{2}{ }^{\prime} \cdot \omega_{1}{ }^{\prime} \cdot \omega_{4}{ }^{\prime}\right)+\left(48 \omega_{2}{ }^{\prime} \cdot \omega_{1}{ }^{\prime 2} \cdot \omega_{4}{ }^{\prime}\right)-\left(128 \omega_{1}{ }^{\prime} \cdot \omega_{3}{ }^{\prime} \cdot \omega_{4}{ }^{\prime}\right)-\left(64 \omega_{2}{ }^{\prime} \cdot \omega_{3}{ }^{\prime} \cdot \omega_{4}{ }^{\prime}\right)+\left(64 \omega_{2}{ }^{\prime} \cdot \omega_{1}{ }^{\prime}\right)-\left(32 \omega_{2}{ }^{\prime} \cdot \omega_{1}{ }^{\prime 2}\right)$ $+\left(32 \omega_{1}{ }^{\prime}\right)+\left(96 \omega_{2}{ }^{\prime} \cdot \omega_{1}{ }^{\prime} \cdot \omega_{3}{ }^{\prime} \cdot \omega_{4}{ }^{\prime}\right)-\left(32 \omega_{2}{ }^{\prime} \cdot \omega_{1}{ }^{\prime 2} \cdot \omega_{3}{ }^{\prime} \cdot \omega_{4}{ }^{\prime}\right)+\left(64 \omega_{1}{ }^{\prime} \cdot \omega_{3}{ }^{\prime}\right)+\left(32 \omega_{2}{ }^{\prime} \cdot \omega_{3}{ }^{\prime}\right)-\left(32 \omega_{1}{ }^{\prime 2} \cdot \omega_{3}{ }^{\prime}\right)+\left(128 \omega_{1}{ }^{\prime} \cdot \omega_{4}{ }^{\prime}\right)+\left(64 \omega_{2}{ }^{\prime} \cdot \omega_{4}{ }^{\prime}\right)+\left(64 \omega_{3}{ }^{\prime} \cdot \omega_{4}{ }^{\prime}\right)-(64$ $\left.\omega_{1}{ }^{\prime 2} \cdot \omega_{4}{ }^{\prime}\right)+\left(48 \omega_{1}{ }^{\prime 2} \cdot \omega_{3}{ }^{\prime} \cdot \omega_{4}{ }^{\prime}\right)+\left(32 \omega_{4}{ }^{\prime 2}\right)-\left(64 \omega_{4}{ }^{\prime 2} \cdot \omega_{1}{ }^{\prime}\right)+\left(24 \omega_{4}{ }^{\prime 2} \cdot \omega_{1}{ }^{\prime 2}\right)-\left(32 \omega_{4}{ }^{\prime 2} \cdot \omega_{1}{ }^{\prime} \cdot \omega_{2}{ }^{\prime} \cdot \omega_{3}{ }^{\prime}\right)+\left(10 \omega_{4}{ }^{\prime 2} \cdot \omega_{1}{ }^{\prime 2} \cdot \omega_{2}{ }^{\prime} \cdot \omega_{3}{ }^{\prime}\right)+\left(48 \omega_{4}{ }^{\prime 2} \cdot \omega_{1}{ }^{\prime} \cdot \omega_{2}{ }^{\prime}\right)-\left(16 \omega_{4}{ }^{\prime 2} \cdot\right.$ $\left.\omega_{1}^{\prime 2} \cdot \omega_{2}{ }^{\prime}\right)-\left(32 \omega_{4}^{\prime 2} \cdot \omega_{2}{ }^{\prime}\right)-\left(32 \omega_{4}^{\prime 2} \cdot \omega_{3}{ }^{\prime}\right)+\left(48 \omega_{4}^{\prime 2} \cdot \omega_{1}{ }^{\prime} \cdot \omega_{3}{ }^{\prime}\right)-\left(16 \omega_{4}^{\prime 2} \cdot \omega_{1}^{\prime 2} \cdot \omega_{3}{ }^{\prime}\right)+\left(24 \omega_{4}{ }^{\prime 2} \cdot \omega_{2}{ }^{\prime} \cdot \omega_{3}{ }^{\prime}\right)$

$\mathrm{a}[10] 64-\left(16 \omega_{2}{ }^{\prime} \cdot \omega_{1}{ }^{\prime} \cdot \omega_{3}{ }^{\prime}\right)+\left(4 \omega_{2}{ }^{\prime} \cdot \omega_{1}{ }^{\prime 2} \cdot \omega_{3}{ }^{\prime}\right)-\left(32 \omega_{2}{ }^{\prime} \cdot \omega_{1}{ }^{\prime} \cdot \omega_{4}{ }^{\prime}\right)+\left(8 \omega_{2}{ }^{\prime} \cdot \omega_{1}{ }^{2} \cdot \omega_{4}{ }^{\prime}\right)-\left(32 \omega_{1}{ }^{\prime} \cdot \omega_{3}{ }^{\prime} \cdot \omega_{4}{ }^{\prime}\right)-\left(16 \omega_{2}{ }^{\prime} \cdot \omega_{3}{ }^{\prime} \cdot \omega_{4}{ }^{\prime}\right)-\left(64 \omega_{1}{ }^{\prime}\right)-\left(32 \omega_{2}{ }^{\prime}\right)-\left(32 \omega_{3}{ }^{\prime}\right)-(64$ $\left.\omega_{4}{ }^{\prime}\right)+\left(32 \omega_{2}{ }^{\prime} \cdot \omega_{1}{ }^{\prime}\right)-\left(8 \omega_{2}{ }^{\prime} \cdot \omega_{1}{ }^{2}\right)+\left(16 \omega_{1}{ }^{\prime 2}\right)+\left(16 \omega_{2}{ }^{\prime} \cdot \omega_{1}{ }^{\prime} \cdot \omega_{3}{ }^{\prime} \cdot \omega_{4}{ }^{\prime}\right)-\left(4 \omega_{2}{ }^{\prime} \cdot \omega_{1}{ }^{\prime 2} \cdot \omega_{3}{ }^{\prime} \cdot \omega_{4}{ }^{\prime}\right)+\left(32 \omega_{1}{ }^{\prime} \cdot \omega_{3}{ }^{\prime}\right)+\left(16 \omega_{2}{ }^{\prime} \cdot \omega_{3}{ }^{\prime}\right)-\left(8 \omega_{1}{ }^{\prime 2} \cdot \omega_{3}{ }^{\prime}\right)+\left(64 \omega_{1}{ }^{\prime} \cdot \omega_{4}{ }^{\prime}\right)+(32$ $\left.\omega_{2}{ }^{\prime} \cdot \omega_{4}{ }^{\prime}\right)+\left(32 \omega_{3}{ }^{\prime} \cdot \omega_{4}{ }^{\prime}\right)-\left(16 \omega_{1}{ }^{\prime 2} \cdot \omega_{4}{ }^{\prime}\right)+\left(8 \omega_{1}{ }^{\prime 2} \cdot \omega_{3}{ }^{\prime} \cdot \omega_{4}{ }^{\prime}\right)+\left(16 \omega_{4}{ }^{\prime 2}\right)-\left(16 \omega_{4}{ }^{\prime 2} \cdot \omega_{1}{ }^{\prime}\right)+\left(4 \omega_{4}{ }^{\prime 2} \cdot \omega_{1}{ }^{\prime 2}\right)-\left(4 \omega_{4}{ }^{\prime 2} \cdot \omega_{1}{ }^{\prime} \cdot \omega_{2}{ }^{\prime} \cdot \omega_{3}{ }^{\prime}\right)+\left(\omega_{4}{ }^{\prime 2} \cdot \omega_{1}{ }^{\prime 2} \cdot \omega_{2}{ }^{\prime} \cdot \omega_{3}{ }^{\prime}\right)+\left(8 \omega_{4}{ }^{\prime 2} \cdot\right.$ $\left.\omega_{1}{ }^{\prime} \cdot \omega_{2}{ }^{\prime}\right)-\left(2 \omega_{4}{ }^{\prime 2} \cdot \omega_{1}{ }^{\prime 2} \cdot \omega_{2}{ }^{\prime}\right)-\left(8 \omega_{4}{ }^{\prime 2} \cdot \omega_{2}{ }^{\prime}\right)-\left(8 \omega_{4}{ }^{\prime 2} \cdot \omega_{3}{ }^{\prime}\right)+\left(8 \omega_{4}{ }^{\prime 2} \cdot \omega_{1}{ }^{\prime} \cdot \omega_{3}{ }^{\prime}\right)-\left(2 \omega_{4}{ }^{\prime 2} \cdot \omega_{1}{ }^{\prime 2} \cdot \omega_{3}{ }^{\prime}\right)+\left(4 \omega_{4}{ }^{\prime 2} \cdot \omega_{2}{ }^{\prime} \cdot \omega_{3}{ }^{\prime}\right)$

\begin{tabular}{cl}
\hline $\mathrm{b}[0]$ & $16 \omega_{4}{ }^{2}$ \\
\hline $\mathrm{b}[1]$ & $32 \omega_{4}{ }^{2}$ \\
\hline $\mathrm{b}[2]$ & $-48 \omega_{4}{ }^{\prime 2}$ \\
\hline $\mathrm{b}[3]$ & $-128 \omega_{4}{ }^{\prime 2}$ \\
\hline $\mathrm{b}[4]$ & $32 \omega_{4}{ }^{2}$ \\
\hline $\mathrm{b}[5]$ & $192 \omega_{4}{ }^{\prime 2}$ \\
\hline $\mathrm{b}[6]$ & $32 \omega_{4}{ }^{\prime 2}$ \\
\hline $\mathrm{b}[7]$ & $-128 \omega_{4}{ }^{\prime 2}$ \\
\hline $\mathrm{b}[8]$ & $-48 \omega_{4}{ }^{\prime 2}$ \\
\hline $\mathrm{b}[9]$ & $32 \omega_{4}{ }^{\prime 2}$ \\
\hline $\mathrm{b}[10]$ & $16 \omega_{4}{ }^{\prime 2}$ \\
\hline $\mathrm{G}_{\mathrm{A}}$ & $10^{(2 / 20)}$
\end{tabular}

The normalization constant $\mathrm{G}_{\mathrm{A}}$ is used to provide $0 \mathrm{~dB}$ gain at $1 \mathrm{kHz}$. 
data acquisition process is the selection of an appropriate sampling rate. The Nyquist-Shannon sampling theorem states that the sampling frequency should not be less than twice the maximum frequency that is to be recorded ${ }^{10)}$. For time-domain analysis higher sample rates are desirable due to waveform distortion that occurs as the frequency of the signal of interest approaches the Nyquist frequency. The actual sample rates required to meet the standards are discussed later in the paper.

With a digital filter it is possible to modify the frequency and phase response of a system by applying a series of multiplications and additions to the time-domain data (for a detailed description of digital filtering see Oppenheim and Schafer ${ }^{11)}$ or Rabiner and Gold ${ }^{12)}$ ). There are essentially two methods of filtering a signal digitally: Finite Impulse Response (FIR) and Infinite Impulse response (IIR). FIR filters work by multiplying the current and previous data samples by a set of coefficients and summing the results. The value of the current output sample, $y[n]$, is given by:

$y[n]=\sum_{k=0}^{M} b_{k} \cdot x[n-k]$

where $b_{k}$ is the $k^{\text {th }}$ filter coefficient, $x[n-1]$ is the previous input sample and $\mathrm{M}$ is the filter order. An IIR filter uses previous output values in addition to previous input values to calculate the current output sample value, which may be given by:

$$
y[n]=\frac{1}{a_{0}}\left[\sum_{j=0}^{M} b_{j} \cdot x[n-j]-\sum_{k=1}^{M} a_{k} \cdot y[n-k]\right]
$$

Whilst FIR filters have some advantages (for example, they are always stable and they can be designed to have linear phase), their main disadvantage is that they would need a large number of coefficients (possibly thousands) to match the frequency magnitude response of the weighting filters given in the standards. As will be seen later, the IIR $\mathrm{A}$-weighting and $\mathrm{C}$-weighting filters only require twenty two and fourteen coefficients respectively. As the number of coefficients are small, it is convenient to publish generic equations that are a function of sampling frequency for each coefficient. It is possible that a poor IIR filter design could be unstable (due to the recursive nature of the filter); however, the filters presented here are stable.

\section{Bilinear Transform}

There are a number of methods for deriving a digital fil- ter from an analogue s-domain filter. One possible method is to convert the analogue (s-domain) impulse response given in ANSI S1.42 to a digital (z-domain) impulse response using the Impulse Invariance method; however, this is not straight forward because the selection of sample rate needs to account for aliasing in the frequency domain. In this paper, the authors propose the bilinear transform method with frequency warping as it maps the entire $j \omega$ axis in the s-plane to one revolution of the unit circle in the $z$-plane ${ }^{11)}$ which eliminates the aliasing problem. In the bilinear transform method of digital IIR filter design, ' $s$ ' in the analogue s-domain equation is replaced by the bilinear transform defined by Equation $5^{12)}$.

$s \rightarrow 2 \frac{\left(1-z^{-1}\right)}{\left(1+z^{-1}\right)}$

There is, however, a non-linear relationship between the analogue frequency and the digital frequency. Pre-warping the frequencies used in the analogue s-domain equations (with the substitution shown in Equation 6) can eliminate this problem.

$\omega_{n}^{\prime}=2 \tan \left(\frac{\omega_{n}}{2}\right)$

where $\omega_{n}$ is the normalised filter design frequency (i.e. $2 \pi \omega_{c} / \omega_{s}$ or $2 \pi f_{c} / f_{s}$, where $\omega_{c}$ and $f_{c}$ are the centre frequency and $\omega_{s}$ and $f_{s}$ are the sampling frequency) and $\omega_{n}$ ' is the normalised warped frequency.

To design the equivalent digital IIR filter, Equation 5 is substituted into the analogue s-domain equation, which is simplified until it is in the form of Equation 7 (shown here for a second-order filter, where $M=2$ ). The values for $\omega_{n}$ should then be warped using Equation 6 to give the final filter coefficients. The filter coefficients ( $a_{0}$ to $a_{M}$ and $b_{0}$ to $b_{M}$ ) may then be read directly.

$$
H(z)=\frac{b_{2} z^{-2}+b_{1} z^{-1}+b_{0}}{a_{2} z^{-2}+a_{1} z^{-1}+a_{0}}
$$

\section{Worked example: Low-pass filter}

This section contains a worked example to show the process of designing an IIR digital filter from an analogue s-domain equation. Consider a generic second-order $(M=2)$ low-pass filter:

$$
H(s)=\frac{\omega^{2}}{s^{2}+\frac{\omega}{Q} s+\omega^{2}}
$$




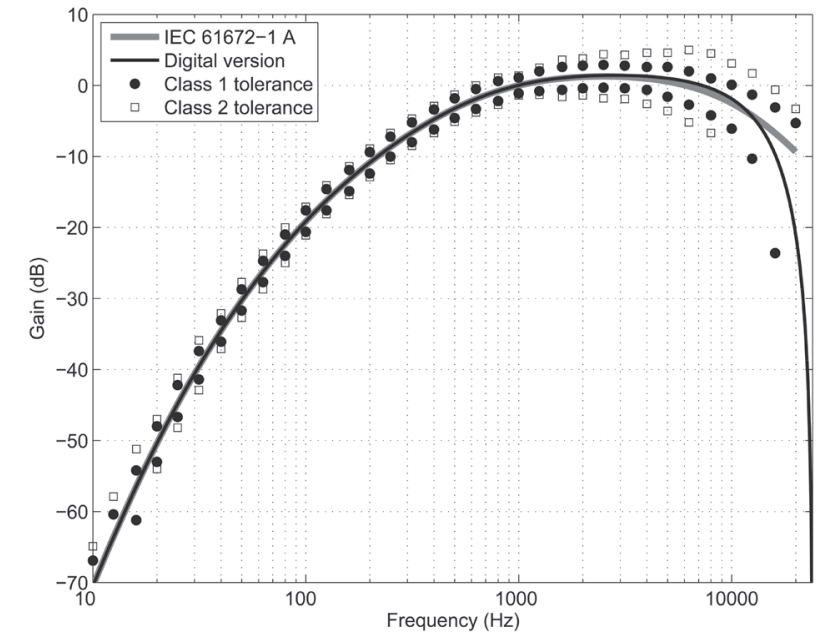

Fig. 2. Digital implementation of the A-weighting filter $(\mathrm{fs}=48 \mathrm{kHz})$

and let $\omega=1570.796 \mathrm{rad} / \mathrm{s}$ (i.e. $f=250 \mathrm{~Hz}$ ), $Q=1 / \sqrt{2}$, and $f_{s}=1000 \mathrm{~Hz}$.

Stage one of the process is to apply the bilinear transform. Substituting Equation 5 into Equation 8 gives:

$$
H(z)=\frac{\omega^{2}}{\left[2 \frac{\left(1-z^{-1}\right)}{\left(1+z^{-1}\right)}\right]^{2}+\frac{\omega}{Q}\left[2 \frac{\left(1-z^{-1}\right)}{\left(1+z^{-1}\right)}\right]+\omega^{2}}
$$

The second stage of the process is to expand and simplify Equation 9, to place it in the form of Equation 7:

$$
H(z)=\frac{\left[\omega^{2} Q\right] z^{-2}+\left[2 \omega^{2} Q\right] z^{-1}+\left[\omega^{2} Q\right]}{\left[4 Q+\omega^{2} Q-2 \omega\right] z^{-2}+\left[2 \omega^{2} Q-8 Q\right] z^{-1}+\left[4 Q+\omega^{2} Q+2 \omega\right]}
$$

The third stage is to perform the frequency warping procedure. The normalised centre frequency, $\omega n=2 \pi \omega /$ $\omega s=2 \pi f l f s=2 \pi \times 250 / 1000=1.57 \mathrm{rad} / \mathrm{s}$. From Equation 6, the warped frequency is given by $\omega_{n}{ }^{\prime}=2 \tan \left(\frac{\omega_{n}}{2}\right)$.

Thus, the IIR filter coefficients are as follows (where $\omega_{n}{ }^{\prime}=$ 2.0 and $Q=1 / \sqrt{2}$ ):

$$
\begin{aligned}
& b_{2}=\omega_{n}{ }^{\prime 2} Q=2.83 \\
& b_{1}=2 \omega_{n}{ }^{2} Q=5.66 \\
& b_{0}=\omega_{n}{ }^{2} Q=2.83 \\
& a_{2}=4 Q+\omega_{n}{ }^{2} Q-2 \omega_{n}{ }^{\prime}=1.66 \\
& a_{1}=2 \omega_{n}{ }^{2} Q-8 Q=0 \\
& a_{0}=4 Q+\omega_{n}{ }^{2} Q+2 \omega_{n}{ }^{\prime}=9.66
\end{aligned}
$$

Once these six quantities have been calculated, they can

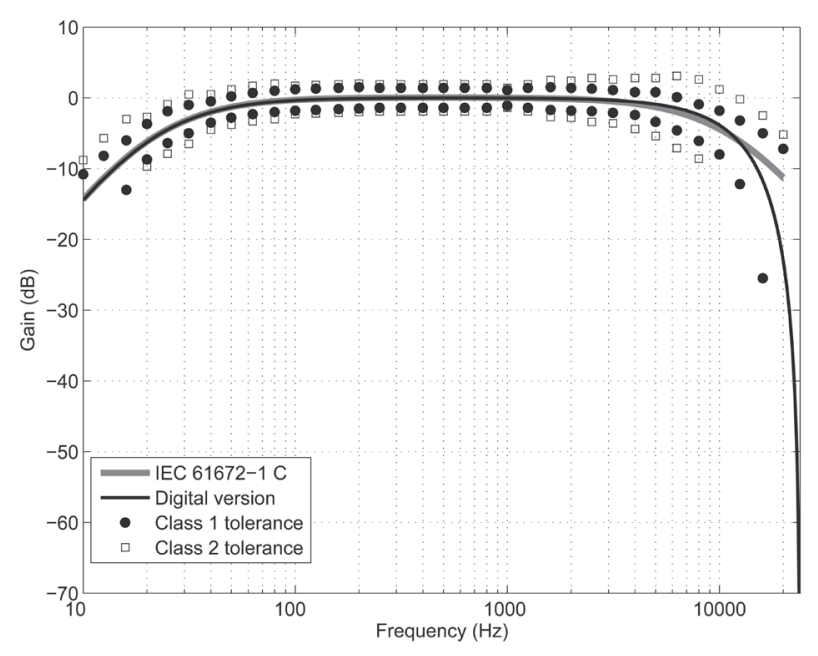

Fig. 3. Digital implementation of the $\mathrm{C}$-weighting filter $(\mathrm{fs}=48 \mathrm{kHz})$

be used with Equation 4 in order to calculate the filtered signal. Equation 4 may be expanded as follows for this example:

$$
y[n]=\frac{1}{a_{0}}\left(b_{0} x[n]+b_{1} x[n-1]+b_{2} x[n-2]-a_{1} y[n-1]-a_{2} y[n-2]\right)
$$

\section{IIR Digital Weighting Filter Coefficients}

By applying the bilinear transform to Equations 1 or 2 , it is possible to design the IIR weighting filter with the corresponding frequency magnitude response. The general form for an IIR filter section is given by Equation 7, where $a_{\mathrm{k}}$ to $b_{\mathrm{j}}$ are the coefficients defined in Tables 1 and 2. The values of $\omega^{\prime}$ from these tables are obtained by warping the normalised values of $\omega$ (from Equations 1 and 2) using Equation 6.

For example, $\omega_{1}{ }^{\prime}=2 \tan \frac{\left(2 \pi \frac{f_{1}}{f_{s}}\right)}{2}=2 \tan \left(\pi \frac{f_{1}}{f_{s}}\right)$.

As stated previously, it is necessary to specify a minimum sample rate in order for the filters to meet the tolerances specified in the Standards. Figures 2 and 3 show the frequency magnitude responses of the $\mathrm{A}$ - and $\mathrm{C}$-weighting filters designed using a sample-rate of $48 \mathrm{kHz}$ and with the coefficients calculated according to Tables 1 and 2, respectively. The frequency magnitude responses are calculated as follows:

$|H(f)|=\operatorname{mag}(F F T\{h[n]\})$ 
Table 2. Filter coefficients for the $\mathbf{C}$-weighting filter

\begin{tabular}{|c|c|}
\hline $\mathrm{a}[0]$ & $\left(16 \omega_{4}{ }^{\prime}\right)+\left(16 \omega_{1}{ }^{\prime}\right)+\left(4 \omega_{1}{ }^{\prime 2}\right)+\left(16 \omega_{4}{ }^{\prime} \cdot \omega_{1}{ }^{\prime}\right)+\left(4 \omega_{4}{ }^{\prime} \cdot \omega_{1}{ }^{2}\right)+\left(\omega_{4}{ }^{2} \cdot \omega_{1}{ }^{2}\right)+\left(4 \omega_{4}{ }^{\prime 2)}+16+\left(4 \omega_{4}{ }^{\prime 2} \cdot \omega_{1}{ }^{\prime}\right)\right.$ \\
\hline $\mathrm{a}[1]$ & $\left(8 \omega_{4}^{\prime 2}\right)-32+\left(6 \omega_{4}^{\prime 2} \cdot \omega_{1}^{\prime 2}\right)+\left(32 \omega_{4}^{\prime} \cdot \omega_{1}^{\prime}\right)+\left(16 \omega_{4}^{\prime} \cdot \omega_{1}^{\prime 2}\right)+\left(8 \omega_{1}^{\prime 2}\right)+\left(16 \omega_{4}^{\prime 2} \cdot \omega_{1}^{\prime}\right)$ \\
\hline $\mathrm{a}[2]$ & $-16+\left(20 \omega_{4}{ }^{\prime} \cdot \omega_{1}{ }^{\prime 2}\right)-\left(48 \omega_{4}{ }^{\prime}\right)-\left(4 \omega_{4}{ }^{\prime 2}\right)-\left(4 \omega_{1}{ }^{2}\right)+\left(15 \omega_{4}{ }^{\prime 2} \cdot \omega_{1}{ }^{\prime 2}\right)-\left(48 \omega_{1}{ }^{\prime}\right)+\left(20 \omega_{4}{ }^{\prime 2} \cdot \omega_{1}{ }^{\prime}\right)-\left(16 \omega_{4}{ }^{\prime} \cdot \omega_{1}{ }^{\prime}\right)$ \\
\hline $\mathrm{a}[3]$ & $64-\left(16 \omega_{1}^{\prime 2}\right)-\left(64 \omega_{4}^{\prime} \cdot \omega_{1}^{\prime}\right)+\left(20 \omega_{4}^{\prime 2} \cdot \omega_{1}^{\prime 2}\right)-\left(16 \omega_{4}^{\prime 2}\right)$ \\
\hline $\mathrm{a}[4]$ & $-\left(4 \omega_{1}{ }^{\prime 2}\right)+\left(15 \omega_{4}{ }^{2} \cdot \omega_{1}{ }^{\prime 2}\right)-\left(4 \omega_{4}{ }^{\prime 2}\right)+\left(48 \omega_{1}{ }^{\prime}\right)-\left(16 \omega_{4}{ }^{\prime} \cdot \omega_{1}{ }^{\prime}\right)-\left(20 \omega_{4}{ }^{\prime} \cdot \omega_{1}{ }^{2}\right)-16-\left(20 \omega_{4}{ }^{\prime 2} \cdot \omega_{1}{ }^{\prime}\right)+\left(48 \omega_{4}{ }^{\prime}\right)$ \\
\hline $\mathrm{a}[5]$ & $\left(8 \omega_{4}^{\prime 2}\right)-\left(16 \omega_{4}^{\prime 2} \cdot \omega_{1}^{\prime}\right)+\left(8 \omega_{1}^{\prime 2}\right)+\left(32 \omega_{4}^{\prime} \cdot \omega_{1}^{\prime}\right)-\left(16 \omega_{4}^{\prime} \cdot \omega_{1}^{\prime 2}\right)+\left(6 \omega_{4}^{\prime 2} \cdot \omega_{1}^{\prime 2}\right)-32$ \\
\hline $\mathrm{a}[6]$ & $-\left(4 \omega_{4}{ }^{\prime} \cdot \omega_{1}{ }^{\prime 2}\right)+\left(16 \omega_{4}{ }^{\prime} \cdot \omega_{1}{ }^{\prime}\right)+\left(\omega_{4}{ }^{\prime 2} \cdot \omega_{1}{ }^{\prime 2}\right)-\left(4 \omega_{4}{ }^{2} \cdot \omega_{1}{ }^{\prime}\right)+16-\left(16 \omega_{1}{ }^{\prime}\right)-\left(16 \omega_{4}{ }^{\prime}\right)+\left(4 \omega_{4}{ }^{2}\right)+\left(4 \omega_{1}{ }^{\prime 2}\right)$ \\
\hline $\mathrm{b}[0]$ & $4 \omega_{4}^{\prime 2}$ \\
\hline $\mathrm{b}[1]$ & $8 \omega_{4}^{\prime 2}$ \\
\hline $\mathrm{b}[2]$ & $-4 \omega_{4}{ }^{2}$ \\
\hline $\mathrm{b}[3]$ & $-16 \omega_{4}^{\prime 2}$ \\
\hline $\mathrm{b}[4]$ & $-4 \omega_{4}^{\prime 2}$ \\
\hline $\mathrm{b}[5]$ & $8 \omega_{4}^{\prime 2}$ \\
\hline $\mathrm{b}[6]$ & $4 \omega_{4}{ }^{2}$ \\
\hline $\mathrm{G}_{\mathrm{C}}$ & $10^{(-0.062 / 20)}$ \\
\hline
\end{tabular}

The normalization constant, $\mathrm{G}_{\mathrm{C}}$, is used to provide $0 \mathrm{~dB}$ gain at $1 \mathrm{kHz}$.

Where FFT \{\} is a real to complex Fast Fourier Transform, $\operatorname{mag}()$ is the magnitude of a complex number and $h[n]$ is the impulse response of the filter calculated from Equation 4 where:

$$
\begin{aligned}
& h[n] \equiv y[n] \\
& x[n]=1, n=0 \\
& x[n]=0, n>0
\end{aligned}
$$

As the impulse response of the filter decays to zero, it is not necessary to use a windowing function. This method of calculating the frequency response of a digital filter is similar to the freqz () function in Matlab ${ }^{\circledR}$.

Also shown in Figs. 2 and 3 are the weighting curves and the Class $1 \&$ Class 2 tolerances presented in IEC 61672-1. From Figs. 2 and 3 it may be seen that the digital filter presented here meets the standard of the Class 1 filter. Although the specification of the A- and C-weighting curves is the same in both IEC 61672-1 and ANSI S.143 , the tolerances are different. IEC 61672-1 defines two sets of tolerances: Class 1 and Class 2; whereas, ANSI S.1-43 defines three sets of tolerances: Type 0 , Type 1 and Type 2. Table 3 shows the minimum sample rate required to meet the full tolerances specified in the standards. Note that this is the sample rate of the filter and not necessarily that of the recorded data, as the recorded data could be resampled before being filtered.

\section{Conclusions}

Many workers are exposed to noise in their working environment. Excessive noise exposure can cause health problems and, therefore, it is important that the worker's
Table 3. Minimum sampling frequency required to meet the full tolerances specified in IEC 61672-1 and ANSI S.1-43

\begin{tabular}{lcc}
\hline & A-weighting & C-weighting \\
\hline IEC 61672-1 Class 1 & $35 \mathrm{kHz}$ & $35 \mathrm{kHz}$ \\
IEC 61672-1 Class 2 & $20 \mathrm{kHz}$ & $20 \mathrm{kHz}$ \\
ANSI S.1-43 Type 0 & $71 \mathrm{kHz}$ & $72 \mathrm{kHz}$ \\
ANSI S.1-43 Type 1 & $35 \mathrm{kHz}$ & $35 \mathrm{kHz}$ \\
ANSI S.1-43 Type 2 & $20 \mathrm{kHz}$ & $20 \mathrm{kHz}$ \\
\hline
\end{tabular}

noise exposure is measured and/or monitored by the equipment manufacturer or by the employer. Human exposure to noise may be measured using microphones; however, weighting filters are also required to correlate the physical noise measurements to the perceived loudness of the noise. The weighting filters are presented in the relevant standards (IEC 61672-1 and ANSI S1.43), but not in a format that can be implemented directly as a digital filter. This paper has presented a method of implementing the frequency weightings as digital filters, which can be used with any numerical processing software (such as a spreadsheet or programming language running on a $\mathrm{PC}$, mobile device or embedded system). Weighting filters were generated using the method described in this paper and the frequency responses compared to those given in the standards and were thus verified to be within the allowable tolerances. Using the information presented, it is possible to design an IIR digital filter of arbitrary sample rate to weight the recorded time-domain noise SPL data, necessary for calculating the A- or C-weighted exposure and thus the maximum healthrelated exposure times in accordance with the international 
standards and the EU Directive.

\section{References}

1) European Commission (2003) Directive 2003/10/EC of the European Parliament and of the Council of 6th February 2003 on the minimum health and safety requirements regarding exposure of workers to the risks arising from physical agents (noise). Off J Eur Communities L42, 38.

2) Her Majesty's Stationery Office (HMSO) (2005) The Control of Noise at Work Regulations 2005. Statutory Instrument 2005 No. 1643. HMSO, Norwich, UK.

3) ISO 226 (2003) Acoustics - Normal equal-loudness-level contours. International Organization for Standardization (ISO), Geneva.

4) IEC 61672-1 (2002) Electroacoustics —-Sound level meters — Part 1: Specifications. International Electrotechnical Commission (IEC), Geneva.

5) ANSI S1.43 (1997, R 2007) Specifications for IntegratingAveraging Sound Level Meters. American National Standards Institute (ANSI) / Acoustical Society of America (ASA), New York.

6) ISO 4869-2 (1995) Acoustics - Hearing protectors-
Part 2: Estimation of effective A-weighted sound pressure levels when hearing protectors are worn. International Organization for Standardization (ISO), Geneva.

7) Rimell AN, Mansfield NJ (2007) Design of digital filters for frequency weightings required for risk assessments of workers exposed to vibration. Ind Health 45, 512-9. [Medline] [CrossRef]

8) Rimell A, Mansfield NJ (2007) Design of digital filters for frequency weightings required for risk assessments of workers exposed to vibration-corrigendum. Ind Health 48, 743. [CrossRef]

9) ANSI S1.42 (2001, R 2011) Design Response of Weighting Networks for Acoustical Measurements. American National Standards Institute (ANSI) / Acoustical Society of America (ASA), New York.

10) Shannon C (1949) Communication in the Presence of Noise. Proc IRE 37, 10-21 (Republished in Proceedings of the IEEE, 1998, 86, 447-57). [CrossRef]

11) Oppenheim A, Schafer R (1989) Discrete-time Signal Processing, Prentice-Hall, Upper Saddle River, New Jersey.

12) Rabiner LR, Gold B (1975) Theory and Application of Digital Signal Processing, Prentice-Hall, Upper Saddle River, New Jersey. 